\title{
Archéologie chez les Taïpi. Hatiheu, un projet partagé aux îles Marquises, de Pierre Ottino-Garanger
}

\section{Gilles Bounoure}

\section{OpenEdition}

1 Journals

\section{Édition électronique}

URL : http://journals.openedition.org/jso/5836

DOI : $10.4000 /$ jso.5836

ISSN : 1760-7256

\section{Éditeur}

Société des océanistes

\section{Édition imprimée}

Date de publication : 30 juin 2009

Pagination : $175-176$

ISBN : 978-2-85430-024-6

ISSN : 0300-953x

Référence électronique

Gilles Bounoure, "Archéologie chez les Taïpi. Hatiheu, un projet partagé aux îles Marquises, de Pierre Ottino-Garanger », Journal de la Société des Océanistes [En ligne], 128 I janvier-juin 2009, mis en ligne le 29 avril 2009, consulté le 22 septembre 2020. URL : http://journals.openedition.org/jso/5836 ; DOI : https://doi.org/10.4000/jso.5836 
égards bien cruelle, le grand anthropologue qu'est Pierre Maranda annonce probablement plus que son livre n'en écrit. Ce livre est en effet plus qu'un aveu à la Costa-Gavras ou une confession à la Rousseau. Son récit et son annexe sur les conditions contemporaines d'accès aux lieux et aux personnes des Lau ne font que renforcer la douloureuse impression que l'on retire sur les singulières circonstances de l'ostracisme qu'il est amené à vivre, quarante ans après ses magnifiques « premiers terrains ». Voici un anthropologue qui aura vécu à la fois l'endroit et l'envers d'un même terrain, étant passé d'un accueil extrêmement favorable à une réprobation radicale valant interdiction de séjour.

On doit lui reconnaître une honnêteté respectable à confesser le sort (avec la polysémie très anthropologique qui sied à ce terme) qu'il subit. Je tiens pourtant ce livre pour l'enterrement à la fois symbolique et réel des pratiques anthropologiques des $\mathrm{XIX}^{\mathrm{e}}$ et $\mathrm{XX}^{\mathrm{e}}$ siècles. Il inaugure peut-être même une révolution dans la discipline, qui n'est sans doute pas encore bien perçue par les professionnels, mais dont les signes montent progressivement en puissance jusqu'à l'exacerbation manifeste. De quoi s'agit-il ? Des droits et des devoirs $\mathrm{du}$ « terrain », j'emploie le singulier à dessein.

Il paraît évident à tout anthropologue que le devoir absolu d'avoir à "faire du terrain » s'accompagne symétriquement d'un imprescriptible droit d'accès. Or rien n'est moins sûr, ou ne l'est plus du tout. Malgré toutes les dénégations théoriques de l'auto-proclamée « observation participante » (Godelier, JSO 2007-2), le terrain ethnographique peut apparaître (et transparaît ainsi dans maintes pratiques effectives) comme une forme d'agression. La violence exercée sur terrain adverse n'a d'équivalent que le retour du boomerang. Dans l'expérience de Pierre Maranda, la chance du premier terrain (un providentiel don du ciel et la faveur de mythes historiques que l'on croirait effectivement sortis d'un ouvrage de Marshall Sahlins) n'a d'égale que la malchance de l'après-terrain. La symétrie est parfaite.

Mais on peut aller plus loin que d'en référer à un hasard culturel. Une histoire structurelle est peut-être en train de s'écrire sur le dos de l'anthropologie, indépendamment des anthropologues qui en font les frais. Autrement dit, ce n'est pas tant le comportement personnel de l'anthropologue qui est à mettre en cause, que le principe de la pratique anthropologique ellemême. Il est évident que tous les congrès d'anthropologues qui se sont penchés, depuis les années 1980, sur les nouvelles conditions d'accès au terrain, conditions résultant en particulier des indépendances, sont dans l'obligation non seulement d'entériner les situations de blocage, mais de renouveler leur approche du problème.

La nouveauté est celle-ci : l'accès symétrique, par les populations rencontrées, aux informations scientifiques et la prise de conscience de l'inégalité des statuts entre le chercheur et le " cherché » sont quelques-unes des clés qui permettent de comprendre l'évolution des problèmes et de leurs solutions. La nouvelle formulation des problèmes apporte en même temps une nouvelle forme de solution. Le fait que chaque culture produise maintenant ses universitaires n'a pas encore retenu l'attention des praticiens actuels, et pourtant on doit s'attendre à ce que ses effets soient non seulement locaux, mais bien globaux.

Raymond MAYER,

Université Omar Bongo de Libreville et Université de Lyon 2

\section{McFadgen Bruce, 2007. Hostile Shores. Catastrophic Events in Prehistoric New Zealand and their Impact on Maori Coastal Communities, Auckland Uni- versity Press, Auckland. xiv-298 p., appendices, bibliogr., index, nombreuses ill. noir et blanc, cartes et figures dans le texte.}

Dû à un archéologue chevronné, auteur de plus de soixante-dix contributions scientifiques tirées de quatre décennies de travail de fouille et d'analyse des données de terrain, ce livre a deux ambitions explicites, exposées dans son sous-titre et rappelées p. $15:$ :en premier lieu décrire les diverses catastrophes naturelles » anciennes survenues en Nouvelle-Zélande « dans leur contexte stratigraphique pour corréler et dater ces événements préhistoriques » et « en second lieu explorer les effets de ces événements sur les communautés littorales préhistoriques ». Sur ces deux sujets formant aussi le plan du livre, il apporte à la fois des connaissances de première main et de remarquables exposés synthétisant avec grande clarté les progrès récents de l'archéologie néo-zélandaise. Mais la conclusion qu'il finit par en tirer semblera considérable à quiconque connaît un peu la civilisation et les arts maori, et le caractère encore énigmatique de leur évolution.

On l'oublie trop souvent, la Nouvelle-Zélande reste le théâtre de phénomènes volcaniques et sismiques intenses, du fait de sa situation à la jointure de deux plaques tectoniques, l'indo-australienne et la pacifique, le long d'une zone de subduction que prolongent notamment les fosses sous-marines de Puységur au sud et des Kermadec au nord. À côté de manifestations telluriques spectaculaires et quelquefois terrifiantes rapportées par les voyageurs ou les journalistes, rien n'est plus impressionnant que la carte dressée en 1952 par Harold Wellman (p. 21), montrant, d'après leurs correspondances géologiques, le glissement horizontal sur $480 \mathrm{~km}$ des parties est et ouest de l'île du Sud le long de la «faille alpine» qui la traverse entièrement. L'île du Nord comporte également diverses failles de même orientation, elles aussi à l'origine d'éruptions et de séismes, mais, selon l'auteur, les populations maori d'abord établies sur les côtes avaient moins à craindre de ces phénomènes que des tsunamis engendrés par l'activité tectonique sous-marine, et dont l'archéologie met en évidence les ravages répétés sur le littoral et les estuaires qui le ponctuent.

Voilà pourquoi, dans cette Nouvelle-Zélande qui peut être considérée globalement comme un «lieu précaire de résidence » (titre du premier chapitre), les Maori qui se fixèrent d'abord sur ses rivages empoissonnés, giboyeux et relativement fertiles, en vinrent à s'éloigner de ces côtes devenues « hostiles » au terme 
de catastrophes répétées et qui semblent s'être précipitées aux alentours de l'an 1500 de notre ère. Les faisceaux très serrés d'indices archéologiques, biologiques, chimiques, géologiques sur lesquels Bruce McFadgen appuie ses hypothèses toujours prudentes, en appelant du reste les spécialistes à les discuter fermement (p. XIII), proviennent de plusieurs milliers de sites, et il est évidemment impossible de résumer ici une telle masse de données, ainsi que l'auteur le fait dans ses appendices. Mais, tout comme les recherches de Roger Duff (1950) ont établi sans conteste la réalité de The Moa-hunter Period of Maori Culture, on retiendra certainement comme un apport essentiel à l'archéologie et à l'histoire maori cette leçon de Hostile shores, la mise en évidence de l'abandon massif des sites côtiers au cours $d u X I^{\mathrm{e}}$ siècle, et la réinstallation de leurs descendants dans des zones plus abritées, quoique plus difficiles à aménager, à cultiver et à défendre.

De ce livre souvent très technique mais de lecture aisée et parfois passionnante, on conservera les deux paragraphes extrêmement suggestifs sur lesquels s'achève le corps du texte (p. 237) :

« Le tableau qui se dégage du panorama des catastrophes ici recensées est que, globalement, on pouvait s'arranger des séismes, même sévères, parce que leurs effets étaient généralement localisés, et que les pertes infligées aux populations et aux ressources étaient limitées. Avec l'avancée des dunes littorales, les communautés avaient le temps de s'adapter au fur et à mesure de l'avancée du sable dans les terres. Même dans ces circonstances, l'ensevelissement des jardins et des habitations imposait des ruptures considérables aux habitants des côtes, et forçaient des communautés à s'établir ailleurs. Mais les tsunamis du $x v^{\mathrm{e}}$ siècle introduisirent une situation toute différente. Ils étaient de grande ampleur et durent anéantir complètement les ressources de nourriture. Des gens moururent, soit des blessures provoquées par ces événements, soit des famines qu'ils entraînèrent. Les tsunamis accélèrent une crise que les communautés littorales commençaient tout juste à affronter, la surpopulation et le manque de nourriture. "

«Ainsi, les catastrophes $\mathrm{du} \mathrm{xv}^{\mathrm{e}}$ siècle déclenchèrent un changement culturel en accroissant de manière aiguë la raréfaction des ressources alimentaires. Les tsunamis marquèrent ce changement de leur empreinte, en tuant sélectivement des populations et en détruisant leurs réalisations. Mais ces catastrophes ne furent pas les causes premières du changement, qu'il faut chercher dans les pratiques culturelles des Maori de l'époque archaïque eux-mêmes, prédateurs très efficaces mais beaucoup moins habiles à préserver leurs ressources. Ce sont plutôt ces catastrophes qui ont exacerbé et accéléré des changements déjà en germe et qui étaient très probablement inévitables. »

Cette dernière hypothèse, qui s'appuie notamment sur l'exemple de l'extinction « anthropique » du moa, devra elle aussi être discutée, mais il est précieux de disposer avec ce livre d'un début d'explication de cette énigme, le passage de la culture maori de sa phase archaïque à sa phase classique, si manifeste dans l'ensemble de ses productions artistiques.

\section{RÉFÉRENCE CITÉE}

DuFf Roger, 1950. The Moa-hunter Period of Maori Culture, Wellington, Dept. of Internal Affairs, Canterbury Museum bulletin 1.

Gilles BounOure
Ottino-Garanger Pierre, 2006. Archéologie chez les Taïpi. Hatiheu, un projet partagé aux îles Marquises, $\mathrm{Au}$ Vent des îles-IRD Éditions, Tahiti-Paris, 176 p., bibliogr., annexes, lexique, très nombreuses ill., plans et cartes en couleur.

Servi par un titre évoquant l'aventure, une présentation aérée faisant la part belle aux images et une rédaction évitant les considérations trop techniques, ce plaidoyer pour une "archéologie partagée » est bien fait pour convaincre un public non spécialiste de la nécessité de cette discipline, "souvent considérée comme une sorte de luxe ou de curiosité émanant de pays privilégiés et ne débouchant sur aucune réponse aux demandes et besoins locaux », comme rappelle lucidement l'auteur en conclusion de son texte principal (p. 93). Aux Marquises, ajoute-t-il, l'archéologie est « une nécessité tant économique que sociale, culturelle et identitaire, assurant une liaison entre le passé, le présent et l'avenir, tout en participant à la cohésion sociale et intergénérationnelle. Loin d'être un frein au développement, elle en serait plutôt le garant », justifiant pleinement l'engagement des chercheurs de l'Institut pour la recherche et le développement.

La démonstration qu'en propose Pierre OttinoGaranger a pour cadre la vallée d'Hatiheu et, plus précisément, un ensemble de vestiges architecturaux établis à mi-pente, à un peu moins d'un demikilomètre au sud de la baie du même nom, sur la côte nord-est de Nuku Hiva. Il appuie son plaidoyer sur une argumentation visuelle persuasive, soixante pages d' " images et moments partagés » (pp. 94-155) en forme d'histoire ou de relation de voyage. Ces photographies évoquent successivement l'approche des grandioses paysages marquisiens, la découverte des vestiges et de leurs pétroglyphes, le travail d'identification ( « relèvement») et de fouille, les chantiers de restauration des infrastructures en pierre et de restitution des constructions en matériaux périssables, la réappropriation ou même la transmission des gestes anciens, et les fêtes qu'on peut tenir pour une célébration du résultat de ces efforts collectifs. Détail notable, hormis celles qui concernent le cinquième festival des arts des Marquises, en 1999-2000, les photographies ne sont pas datées et leurs légendes relèvent plus de la narration que de la description. Ce récit en images semble ainsi en appeler davantage à l'émotion qu'à une perception analytique du travail archéologique, de son déroulement, de ses difficultés et de ses enjeux, qui sortait certainement des ambitions de ce livre.

Complété par une intéressante annexe procurant deux versions (l'une plus longue recueillie par Henri Lavondès, la seconde, plus courte, recueillie par l'auteur) de la « légende de Keikahanui », personnage sans doute à l'origine de la construction du paepae Tahakia, sur lequel se sont concentrés les travaux relatés par ce livre, le texte principal s'organise en deux sections. La première (« les racines et le tronc », pp. 671) présente un exposé historique et archéologique consistant, la seconde ( « les branches et le feuillage », pp. 72-93) porte sur l'évolution récente et la situation actuelle de ce territoire de Nuku Hiva, le travail de relevage et de restitution des constructions, et pour 
finir, sur le « renouveau culturel » associé à la mise en pratique de « l'archéologie partagée », avec des photographies préparant les lecteurs au récit en images déjà évoqué, lui-même largement éclairé par ce texte préliminaire.

Il serait abusif d'attendre de cet ouvrage, parfois insuffisamment relu (trois accords fautifs pour la seule colonne de gauche de la p. 170) et souvent allusif (à l'exemple de la page 41 invoquant « des témoignages du début du XIX ${ }^{\mathrm{e}}$ siècle » sans autre justification), un état complet des recherches archéologiques menées jusqu'en 2006 dans cette vallée. On peut également regretter de ne pas retrouver sur les plans, par ailleurs fort bien conçus, certains vestiges cités par le texte ou les légendes (« paepae 35 du me'ae Teiipoka » pp. 105106, absent des plans pp. 46-47 et 50-51, sauf à corriger « paepae 15 », paepae $n^{\circ} 195$, pp. 102-104 et $n^{\circ} 39$ de Kamuhei, p. 107 non repérés sur le plan p. 37). «Nombre d'études ne sont pas terminées, comme d'ailleurs une partie des travaux de terrain », indique l'auteur (p. 45) et il va de soi que, dans un volume si largement illustré, son texte ne pouvait résumer que de manière sommaire ou allusive les observations et les recherches nombreuses auxquelles ce site a donné lieu depuis plus d'un siècle.

L'ouvrage cite ou résume utilement les plus récentes. Certaines plus anciennes auraient pu être invoquées en défense de "l'archéologie partagée », à l'exemple du surprenant récit laissé par Stevenson de sa visite des vestiges sous la conduite du P. Siméon Delmas (chapitre XI des Mers $d u S u d$ ), ou de ce commentaire de von den Steinen, à propos des anciennes constructions marquisiennes :

« L'impression la plus durable que j'aie reçue était relative à l'étendue du Marae Te Ipoka de Hatiheu sur la côte nord de Nukuhiva, où habitaient des Taipi. Remarquables étaient quelques entassements de pierre comme le $\mathrm{n}^{\circ} 6$. On devait y pratiquer des sacrifices humains de Hapaá, envoyés par ces derniers pour sceller la fin des hostilités, puisque les Taipi avaient donné la même garantie aux Hapaá pour leurs Marae. On s'envoyait des otages pour les mettre à mort ! » (1928:71)

Ces vieilles pages font mesurer tout le progrès accompli entre le regard archéologique traditionnel, où percent mélancolie et autres sentiments intimes, et cette « archéologie partagée » tournée vers le présent et l'avenir collectifs, telle que la défend et la promeut ici l'auteur, dans le droit-fil des idées de José Garanger.

\section{RÉFÉRENCE CITÉE}

Von Den SteInen Karl, 1928. Die Marquesaner und ihre Kunst, Band II, Plastik, Berlin, D. Reimer.

Gilles BounOuRE 